\title{
Légitimation des savoirs environnementaux dans un programme de recherche participative au Sénégal
}

\author{
Frédérique Jankowski ${ }^{1}$, Joëlle Le Marec ${ }^{2}$ \\ 1 Anthropologue, UPR Green, CIRAD, 34398 Montpellier cedex, France \\ 2 Sciences de l'information et de la communication, CERILAC, Université Paris 7 Diderot, 75013 Paris, France
}

Contrairement à une vision naïve, l'échange de savoirs entre scientifiques impliqués et acteurs locaux n'échappe pas à des rapports de pouvoir. L'appropriation des savoirs n'est pas un processus purement cognitif, il est aussi social et politique. Il engage des intermédiaires parfois surprenants. La recherche impliquée - où les chercheurs tentent de produire un savoir situé, spécifique à l'espace/temps du monde local - se trouve aussi confrontée à l'exigence de justifier la généralité de ses méthodes et des résultats que la politique scientifique impose. Cet article pose symétriquement la question de la traduction du savoir savant et celle de la reconnaissance, par la Science, des particularités qui rendent le savoir efficace et pertinent.

La Rédaction

\section{Mots-clés :}

Savoirs académiques et non académiques ; légitimation ; recherche ; gouvernance ; Sénégal
Résumé - La participation est devenue une injonction de modernisation d'une recherche intégrée à des enjeux économiques et sociaux. De précédentes études ont décrit la manière dont la prise en compte des savoirs locaux dans le cadre de recherches participatives implique des remaniements de ces savoirs pour qu'ils répondent à des critères de scientificité. Cet article se propose d'observer les processus de légitimation des savoirs locaux à l'œuvre non pas a posteriori mais au cours d'une recherche participative. Les modes de légitimation des savoirs scientifiques sont également questionnés. Il s'agit d'observer le processus dialectique entre définition de savoirs et rapports de légitimité dans les recherches participatives. Le lien entre processus de légitimation et réappropriation des savoirs issus de l'action collective est discuté.

\section{Keywords:}

Academic and non-academic knowledge; legitimation; research; governance; Senegal

\begin{abstract}
Legitimation of environmental knowledge in a participative research project in Senegal. Participation has become one of the key technical and political standards in the modernization of research embedded in economic and social issues. Previous studies have described how the incorporation of local knowledge in participative research implies reorganizing this knowledge to comply with scientificity criteria. Our paper analyses the legitimation processes of local knowledge not a posteriori, but in the course of a participative research project. The study shows that, in this context, what is being legitimized is not local knowledge only, but that scientific knowledge also becomes a legitimation issue for different actors. Based on data collected between April 2009 and April 2011 during a participative research project in Senegal, we describe various forms of legitimation of local and scientific environmental knowledge. This study exposes the dialectical process at work during the participative research between definitions of knowledge and positioning of power. More broadly, we discuss the link between the legitimation process and the appropriation of the knowledge shared in collective action for both the local and the scientific circles involved.
\end{abstract}

\section{Introduction}

Depuis deux décennies, les démarches participatives dominent le développement rural et la gestion des ressources renouvelables. Si la science a longtemps été considérée comme l'unique moteur du développement, les échecs successifs d'introduction d'innovations techniques et procédurales dans le milieu rural ont imposé une remise en question des méthodes classiques de transfert de technologies et de gestion de la nature et

Auteur correspondant : F. Jankowski, frederique.jankowski@cirad.fr 
favorisé le concept de participation dans le domaine du développement rural. Jusqu'à aujourd'hui, celle-ci recouvre de multiples formes, niveaux d'implications et objectifs (Chambers et al., 1989 ; Oakley, 1991 ; LavigneDelville et al., 2000 ; ComMod, 2005). La participation est devenue une injonction à la fois technique et politique de modernisation d'une recherche intégrée à des enjeux économiques et sociaux. En effet, la recherche en partenariat se caractérise par le souci de produire des savoirs qui soient actionnables (Mormont, 2007), c'est-à-dire qui soient légitimes au regard des préoccupations propres des partenaires et qui soient susceptibles de déboucher sur des pistes d'action cohérentes avec les ressources dont ils disposent.

De précédentes études ont décrit la manière dont la prise en compte des savoirs locaux implique des remaniements de ces savoirs pour qu'ils répondent in fine à des critères de scientificité (Sillitoe et al., 2002 ; Agrawal, 2002). Dans ce contexte, leur légitimité est toujours définie par rapport à leur intérêt potentiel pour le développement ou la science. De multiples décontextualisations des savoirs locaux sont effectuées, par rapport à leurs usages, au pool de connaissances dont ils font partie et à leur dynamique propre (Jankowski, 2013). Agrawal (2002) parle de « scientisation » pour désigner les processus de particularisation, validation et généralisation des savoirs locaux pour que ceux-ci accèdent au rang de savoirs vérifiés et utiles.

Nous proposons ici d'observer les processus de légitimation des savoirs locaux à l'œuvre non pas a posteriori, mais au cours d'une recherche participative. La perspective retenue permet de suivre la manière dont des acteurs (qui prennent position par rapport à des savoirs) ou dont des savoirs (élaborés, discutés transformés par des acteurs) deviennent des opérateurs de légitimation les uns pour les autres.

Nous mobilisons une analyse de certaines situations dans lesquelles les acteurs argumentent face à autrui, ce qui transforme des visions collectivement construites (Boltanski et Thevenot, 1991).

À partir de données recueillies, entre avril 2009 et avril 2011, durant une recherche participative menée au Sénégal, on constate plusieurs phénomènes articulés :

- La science intervient dans des processus de légitimation de savoirs locaux, et les savoirs locaux interviennent symétriquement dans la légitimation des savoirs scientifiques.

- Les savoirs scientifiques interviennent dans l'évolution des rapports de légitimité entre différents acteurs $\mathrm{du}$ projet (chercheurs, producteurs, techniciens de laboratoire).

- L'évolution des conceptions de la recherche et des savoirs dans un contexte partenarial peut mettre en jeu les rapports de légitimité entre les différentes équipes scientifiques.

Les processus de légitimation et l'évolution des rapports de légitimité renforcent ou affaiblissent les positionnements voire les appartenances des individus et des groupes au sein des communautés multiples dont ils sont membres (communauté de producteurs, réseau des communautés villageoises, collectif de recherche du programme, communautés disciplinaires nationales et internationales). Ces processus ouvrent un débat, dans le courant de la recherche, sur la nature des savoirs construits en commun et sur la possibilité de les intégrer dans les résultats du programme de recherche. Ils interviennent également dans l'affirmation de différentes conceptions de la recherche et du savoir entre les équipes de chercheurs impliquées.

Il s'agit donc d'observer le processus dialectique entre définition de savoirs et rapports de légitimité à l'œuvre dans les recherches participatives; ce qui permet de s'interroger sur la réappropriation possible des savoirs issus de l'action collective et sur le sens de ces réappropriations, dans les sphères locales et scientifiques.

\section{Le projet de recherche participative : acteurs, dispositifs et savoirs environnementaux}

Le contexte est celui d'un programme de l'Agence nationale de la recherche portant sur la vulnérabilité des sols agricoles sénégalais soumis aux changements climatiques et à l'évolution des pratiques. Il s'agit d'une recherche interdisciplinaire (microbiologie, physicochimie des sols et sciences sociales) et partenariale avec $l^{\prime}$ implication de divers acteurs locaux ${ }^{1}$.

Ce programme fait suite à deux autres projets participatifs mis en œuvre par des chercheurs du laboratoire de microbiologie de Dakar (Sow et Neyra, 2008) et dont l'objectif spécifique était d'impliquer des acteurs locaux dans l'ajustement d'une technique d'amélioration de la qualité des sols agricoles: la technique d'inoculation. Ainsi, les enjeux de la participation entre les acteurs locaux et les scientifiques sont doubles. Le premier est sociotechnique: développer des solutions techniques pour la mise au point et l'appropriation de l'inoculation des plantes. Le second, cognitif, est la construction collective de connaissances sur la qualité des sols.

Des dispositifs de dialogue, de prise de décision et d'action collective ont été construits et observés dans

1 Les cadres locaux de concertation des organisations de producteurs (CLCOP), l'Agence nationale de conseil agricole et rural (Ancar) et le Centre national de concertation des ruraux (CNCR). 
différents espaces: sites d'enquête dans les villages, ateliers délibératifs multipartenariaux, ateliers de travaux pratiques au laboratoire de microbiologie, et parcelles de recherche et de démonstration dans les champs des agriculteurs.

Les savoirs environnementaux partagés dans le cadre de cette recherche participative sont de diverses natures. Les scientifiques situent leurs travaux de recherche dans le contexte global des phénomènes liés au changement climatique. La technique de l'inoculation a été présentée aux agriculteurs comme une méthode permettant de lutter contre les effets de la diminution du taux de précipitations, laquelle est définie comme l'un des principaux facteurs d'appauvrissement des sols (Krasova-Wade et al., 2010). Ce discours reposait sur des présupposés environnementalistes définissant des relations de causalité entre des éléments de l'environnement. Les savoirs scientifiques partagés concernaient aussi la technique de l'inoculation, la diversité et les fonctions des micro-organismes du sol. Le travail ethnographique, quant à lui, avait pour objectif de décrire les formes locales de la connaissance des sols agricoles.

\section{Légitimité rationnelle et processus exogènes de légitimation des savoirs scientifiques}

\section{Légitimité scientifique a priori ?}

Peut-on parler d'une légitimité scientifique a priori ? Celle-ci est-elle établie dans le milieu rural sénégalais où le projet de recherche participative est mis en œuvre? Selon Ela (2007), dans les sociétés africaines actuelles, le rôle et l'utilité de la science sont questionnés par la population locale. Bourguerra (1993) parle de marginalisation des scientifiques en Afrique. Les années 1990 ont vu les institutions académiques se démanteler sans que la recherche disparaisse, reprise bien souvent dans des structures souples : les ONG. Ainsi de puissantes associations se sont substituées aux politiques publiques et ont fait des instituts de recherche de simples prestataires de service (Waast et Gaillard, 2001). Les communautés rurales impliquées dans le programme de recherche participative avaient une longue expérience des projets de développement étatiques, d'ONG nationales et internationales. Les désillusions successives accompagnant ces projets les ont progressivement décrédibilisés du point de vue des populations locales. Ces projets sont perçus comme des possibilités de s'enrichir ou d'accéder à certains moyens matériels et s'inscrivent dans un rapport souvent clientéliste avec les villageois.

Dans le cadre de la recherche participative dont il est question, les scientifiques ne jouissaient pas a priori de la confiance ou d'une légitimité particulière auprès des communautés rurales. Si l'un des enjeux de la participation pour les scientifiques est d'accéder à une meilleure connaissance du milieu, ces derniers ont également conscience qu'il est important, pour la mise en œuvre $\mathrm{du}$ partenariat et le suivi des protocoles expérimentaux in situ, que les acteurs locaux saisissent les modalités d'élaboration de l'inoculum. Aussi, la première année du projet, des représentants des communautés rurales ont été invités au laboratoire de microbiologie de Dakar pour découvrir le travail réalisé par les microbiologistes et plus précisément les étapes nécessaires à la fabrication de l'inoculum. Pour ce faire, des ateliers de travaux pratiques ont été organisés. Les laborantins ont présenté la manière d'utiliser un ensemble d'instruments aux producteurs. Ces derniers, vêtus d'une blouse blanche, ont ensuite expérimenté la manipulation des microscopes, micropipettes, gel de PCR, etc. Ainsi au travers de leur base matérielle et de la pratique, un ensemble de savoirs singuliers et de compétences spécifiques au travail de recherche en microbiologie ont été exposés et appropriés. C'est donc par un déplacement des acteurs locaux dans le lieu de production des savoirs scientifiques que ceux-ci ont fait l'objet d'un premier processus d'appropriation et ont par conséquent acquis une légitimité auprès des participants. Cette légitimité s'est étendue aux représentants de ce savoir. Les techniciens de laboratoire en particulier, membres de l'équipe de recherche, mais qui ont des liens familiaux étroits avec les communautés de producteurs, ont acquis une position particulière de médiateurs susceptibles de représenter à la fois le point de vue des scientifiques pour les producteurs et le point de vue des producteurs pour les scientifiques.

\section{Processus exogènes de légitimation des savoirs scientifiques}

Un élément important apparaît: les principales démarches de légitimation des savoirs scientifiques dans le cadre du projet ne sont pas le fait des chercheurs. Deux acteurs locaux ont joué un rôle crucial dans la légitimation de ces savoirs scientifiques au cours de la démarche participative.

Le premier est un leader associatif paysan, très proche du lignage fondateur du village de Darou Mousty et bénéficiant d'un statut social élevé. Il présente de nombreuses caractéristiques des courtiers en développement que 1'on rencontre au Sénégal (Blundo, 1995 ; Salzbrunn, 1996). Le lignage fondateur du village est celui de Cheikh Amadou Bamba qui a formé la confrérie musulmane la plus influente du Sénégal : le mouridisme. Après Touba, Darou Mousty est la deuxième capitale du mouridisme. La confrérie mouride joue un rôle économique et politique très important au Sénégal (Cruise O’Brien, 1971 ; Copans, 1980 ; Guèye, 2002). Elle représente une communauté extrêmement soudée qui procure les moyens de déplacement et d'ancrage à l'étranger 
à ses membres (Ebin, 1993) qui fournissent ainsi une véritable rente migratoire, puissant agent de développement pour le pays (Moreno Maestro, 2006). Ce leader paysan a plusieurs frères en Europe qui contribuent de manière active à la mise en place de collaborations entre la communauté et des associations des pays d'accueil. L'intérêt public constitue généralement la légitimation ultime des courtiers en développement (Bierschenk et al., 2000). Lors de nos séjours à Darou Mousty, les villageois ont affirmé travailler pour le bien du village. Ce représentant nous a décrit des projets qu'il y avait développés grâce à ses nombreuses relations dans le pays et au-delà de ses frontières. Il tire ainsi une partie de sa légitimité de son contrôle des flux de l'aide au développement, et de sa capacité à sélectionner ceux-ci en cohérence avec les convictions mourides. Sa maîtrise des procédés de négociation et du réseau des opérateurs du développement, reconnue par l'ensemble des villageois, légitime, de fait, le partenariat avec les scientifiques. La légitimation du partenariat légitime par extension les pratiques et discours des chercheurs.

Le contexte religieux est très important au Sénégal. Un jeune marabout a participé directement à la légitimation des savoirs scientifiques du projet. Nous venons de signaler que le pouvoir économique et politique de la communauté mouride au Sénégal est important. Aussi, les marabouts mourides occupent-ils un rôle central dans la société sénégalaise (Moreau, 1982).

Chacune des rencontres entre les partenaires commençait et se clôturait par une prière dont ce jeune marabout avait la charge lorsqu'il était présent. Au début de l'une des premières réunions partenariales, il ouvrit la séance par la lecture d'un ensemble de sourates qui décrivaient, selon lui, ce dont il était question dans le projet :

Sourates 16 - Sourate des abeilles An-Nahl :

[10] : « C'est Lui qui, du ciel, vous envoie de l'eau dont vous faites votre boisson et qui fait croître des pâturages pour vos troupeaux. »

[11] : " Grâce à cette eau, Il fait encore pousser les céréales, les oliviers, les palmiers, les vignes et toutes sortes d'arbres fruitiers. C'est là, en vérité, un signe pour ceux qui savent réfléchir!»

[13] : «Et les multiples choses aux couleurs si variées que Dieu a répandues pour vous sur la Terre ne sont-elles pas un autre signe pour des gens qui raisonnent?»

Sourate 20 - Sourate de Tâ-Hâ :

[6]: «Le souverain des Cieux, de la Terre, des espaces interstellaires et de tout ce qui se trouve dans les profondeurs du sol. »

$\mathrm{Au}$ cours d'entretiens réalisés à la suite de cette rencontre partenariale, le marabout explicite le choix des sourates : les deux premières sourates $(16 / 10 ; 16 / 11)$ permettent de souligner l'importance de l'eau pour l'Homme, et plus spécifiquement pour la végétation et la production agricole. Rappelons que c'est la diminution du taux de précipitations qui est avancée par les scientifiques comme étant responsable, en grande partie, de la baisse de fertilité des sols agricoles. L'une des sourates (20/6) fut choisie pour sa référence directe à « tout ce qui se trouve dans les profondeurs du sol », elle accrédite ainsi, selon lui, la présence de micro-organismes dans le sol. Enfin, deux sourates $(16 / 11 ; 16 / 13)$ évoquent « ceux qui savent réfléchir », « les gens qui raisonnent » comme les chercheurs.

En évoquant ces sourates, le marabout légitime la démarche et les savoirs des scientifiques, et gagne lui-même en autorité. Lors de la rencontre partenariale au cours de laquelle il cite les sourates qui éclairent la signification de la recherche d'un jour nouveau, l'ensemble des participants, chercheurs compris, est passionné par le lien proposé par l'orateur, et attend ses interventions.

\section{Légitimation scientifique des savoirs locaux au cours du processus participatif}

Un processus de légitimation des savoirs locaux dans le cadre de la recherche participative a consisté en leur insertion dans le protocole expérimental appliqué dans les parcelles tests. La première année, les parcelles tests ont été choisies selon des critères définis par les microbiologistes et les physicochimistes du projet. Au terme de cette année, une rencontre réunit les différents acteurs. Les résultats microbiologiques et physicochimiques sont exposés à l'assemblée. Les scientifiques font part de leurs interrogations quant à l'hétérogénéité des résultats observés pour une même communauté rurale. Une explication est avancée par les agriculteurs : les échantillons prélevés dans un même village correspondent à des types de sols différents. Il s'avère avec l'enquête ethnographique que les analyses ont été faites sur des échantillons de sols hétérogènes si on se réfère aux typologies locales des communautés rurales.

Les typologies locales, soigneusement établies avec l'enquête ethnographique et la collecte de sols associée, sont alors mises en relation avec les résultats d'analyses de la microfaune du sol. Une corrélation entre la fréquence de production de nodules par les plantes et les types de sols dégagés par l'enquête ethnographique est démontrée.

Un nouveau protocole d'échantillonnage est alors défini par les chercheurs pour la seconde année du projet et de nouvelles parcelles tests sont choisies par les agriculteurs en tenant compte des types de sols distingués localement. L'adoption des typologies de sols locales a 
donc abouti à la mise en place d'un nouveau protocole expérimental in situ et a transformé les modes d'échantillonnage des chercheurs microbiologistes, pédologues et généticiens.

Cette démarche partenariale et ce protocole élaboré en commun ont ensuite été présentés dans des conférences internationales comme le congrès de l'AABNF (Association africaine pour la fixation biologique de l'azote) en décembre 2010 à Bamako. Ce congrès constitue un espace d'échanges d'expériences et de savoirs scientifiques en matière de recherche sur la biotechnologie adaptée à l'agriculture. Le président du CLCOP et l'agent de l'Ancar de deux communautés rurales ont été invités non seulement à se rendre à cette rencontre scientifique, mais aussi à présenter eux-mêmes le projet participatif et ses résultats. Cette présentation a suscité de nombreux commentaires de l'assemblée. Certains ont mis en doute l'implication réelle des agriculteurs au vu de la qualité scientifique des résultats présentés au congrès. Ces remarques ont renforcé positivement l'unité partenariale et la conviction partagée de la pertinence de la collaboration entre les acteurs locaux et les chercheurs.

Au-delà de la typologie des sols, l'enquête ethnographique a favorisé la reconnaissance du caractère à la fois complexe et systématique des savoirs environnementaux locaux dans la recherche participative. Elle a participé à un intérêt pour la nature de ces savoirs, très audelà de simples énoncés. Au cours de cette recherche, une démarche photo-ethnographique a été développée. L'un des objectifs de cette démarche était de croiser, au travers des séries photographiques, des définitions relatives aux sols agricoles (typologie, usages, vulnérabilité, etc.). Au même titre que les agriculteurs, les techniciens de l'agriculture et les chercheurs, ont été invités à prendre des photographies de ce qu'ils considéraient comme pertinent pour la description et l'analyse des sols agricoles. Cette démarche a abouti à une exposition présentée lors d'une réunion partenariale à laquelle étaient présentes les autorités locales et la presse régionale. Étaient exposés dans un même ensemble, les «observables » pertinents du point de vue des agriculteurs, des techniciens et des scientifiques (microbiologistes, pédologues, cartographes) pour définir la qualité des sols agricoles.

Ce processus de légitimation des savoirs locaux ne relève pas des mêmes mécanismes que ceux que nous avons évoqués en introduction et qui interviennent généralement a posteriori, avec la traduction et la décontextualisation des savoirs qui les rendent appropriables au regard de la recherche. L'inclusion de la typologie locale des sols dans le protocole expérimental des microbiologistes et physicochimistes est intervenue après vérification d'une corrélation statistique entre les fréquences de nodulation et les types locaux de sols. Il est difficile de dire a posteriori si les savoirs locaux auraient été ou non inclus dans le protocole expérimental, et plus fondamentalement, ce qu'ils auraient pu «déranger » $\mathrm{d}^{\prime}$ une démarche de construction de connaissances scientifiques, si cette corrélation n'avait pas été démontrée. Rappelons que la prise en compte des savoirs locaux dans la recherche participative avait été légitimée au congrès de l'AABNF par la reconnaissance de la qualité scientifique des résultats présentés. Mais il s'agissait d'une forme canonique de la communication scientifique : le congrès.

Cependant, l'approche ethnographique avait spécifiquement pour objectif d'éviter la particularisation des savoirs locaux en décrivant l'ensemble des formes locales de la connaissance des sols. Aussi, la démarche photo-ethnographique autour des parcelles tests a été pensée comme un point de coordination, mais surtout comme un point d'ancrage de la déconstruction des définitions et pratiques des différents acteurs impliqués dans la caractérisation et la gestion concertée des sols. Il ne s'agissait donc pas de rechercher un ajustement des catégories indépendamment des lieux, mais bien de comparer des manières d'établir des distinctions opératoires, selon que l'on est producteur avec un enjeu, celui d'obtenir un rendement, ou chercheur essayant d'avoir une certaine universalité des catégories. La légitimité des différentes formes de savoirs a pu ainsi être considérée au regard des enjeux distincts des acteurs impliqués dans le projet.

\section{Savoirs environnementaux et positionnements sociaux}

\section{Des usages des savoirs scientifiques par les acteurs locaux}

Les processus de légitimation des savoirs ont contribué à renforcer les positions sociales de certains acteurs qui en ont été à l'initiative.

Aujourd'hui, le leader paysan se présente lui-même comme un «microbiologiste paysan ». Il maîtrise le discours des scientifiques et sait expliquer en quoi consiste la technique d'inoculation. Selon Cohen et Comaroff (1976), le médiateur est un manipulateur et producteur de sens, dont le but est de «traduire l'inconnu et l'inaccessible dans le familier et le possible ». Le leader paysan en question ne s'est pas véritablement prêté à une traduction du discours des microbiologistes en langage familier. Il exprime davantage sa grande maîtrise de ce langage. En intégrant les scientifiques dans la sphère villageoise, il se positionne comme paysan et intellectuel (Coll, 2000). Au-delà de sa compétence rhétorique, ce leader paysan rend visible ses aptitudes relationnelles, 
organisationnelles et scénographiques (Bierschenk et al., 2000), notamment au cours d'une réunion partenariale dont il a eu en charge l'organisation. Pour l'occasion, il a convoqué les autorités locales, montrant son aptitude à entrer en contact avec le pouvoir administratif et religieux, mais aussi la presse régionale pour rendre plus largement visible son appartenance à l'ensemble des réseaux réunis et faire ainsi la démonstration de son influence dans la communauté.

Ses compétences ont été saluées publiquement par les chercheurs qui le présentaient comme un acteur-clé du partenariat. La position sociale du leader paysan a été renforcée à la fois dans la communauté villageoise et dans la sphère partenariale. Mais il est important de souligner que les intérêts de ce leader paysan ne se réduisent pas à une volonté purement politique d'extension de sa capacité d'action et d'influence. Les liens avec les chercheurs, l'intérêt pour la recherche et pour les connaissances produites, les dimensions cognitives du partenariat sont manifestes.

Le statut de guide spirituel du jeune marabout a également été renforcé au cours de cette recherche. Sa lecture religieuse des enjeux de cette recherche a procédé $\mathrm{d}^{\prime}$ une forme de réappropriation des savoirs scientifiques et donc d'un renforcement de ceux qu'il possédait. Cette réappropriation des savoirs académiques a relevé d'une traduction du discours scientifique en discours religieux, dans le sens de déplacements d'ensembles et de nouvelles interprétations (Latour, 1987). Dans la religion musulmane, les propriétés de l'environnement sont créées et régies par Dieu (Younes, 2003). L’Homme ne peut contrôler les processus de la nature et donc maîtriser durablement l'évolution de celui-ci. Les déplacements effectués au travers de cette traduction peuvent donc s'entendre en termes: (i) de redéfinition des responsabilités : seuls les hommes sont tenus pour responsables de la dégradation des sols, la diminution des précipitations relève de la volonté de Dieu et doit être considérée comme un signe de mauvais usages des ressources de la part des hommes; (ii) de pouvoir d'action et de maîtrise des hommes sur le milieu : si ces derniers peuvent tenter de réparer leurs erreurs, seul Dieu peut décider de restaurer ou non l'état d'équilibre des éléments naturels; et plus largement en termes (iii) de nature de l'expertise : c'est en tant qu'expert de la religion musulmane que le jeune marabout légitime l'expertise des scientifiques. Dans l'étude de la légitimité des guérisseurs traditionnels au Sénégal, Fassin et Fassin (1988) observent que les individus en manque de légitimité traditionnelle sont les plus prompts à souhaiter une légitimité rationnelle. Peut-on penser que c'est le cas du jeune marabout? Celui-ci serait-il à la recherche d'une légitimité rationnelle? Il ne semble pas que cela soit le cas. Ce marabout est l'un des petits-fils du frère de Cheikh Amadou Bamba. Son ascendance lui confère une légitimité traditionnelle importante (Weber, 1959). Au cours du grand $\mathrm{Magal}^{2}$ de Touba, nous avons été invités à rejoindre la natte de ses talibés (disciples) et à lui poser des questions. À l'occasion de cette fête religieuse, une grande exposition sur l'histoire du mouridisme était organisée. Celle-ci présentait notamment la résistance de Cheikh Amadou Bamba aux colons occidentaux. En discutant de cette exposition avec le marabout et ses talibés, ces derniers soulignèrent que ce sont aujourd'hui les toubabs (Européens) qui viennent vers les marabouts pour obtenir des informations et les aider à avancer dans leurs recherches.

$\mathrm{Au}$ travers du processus participatif, le jeune marabout a ainsi renforcé son statut de partenaire-clé pour le projet, mais aussi d'autorité traditionnelle et de guide spirituel au sein de sa communauté villageoise et religieuse.

Dans les deux cas que nous venons de décrire, les savoirs des scientifiques n'ont pas été utilisés dans une visée académique ni même agrotechnique (la restauration des sols). Ils ont été mobilisés dans des contextes religieux ou sociaux par des autorités.

\section{Rapports de légitimité au sein de la communauté interdisciplinaire de chercheurs}

Les positions des chercheurs ont également évolué au sein de leurs propres communautés. Nous n'avons pas fait porter l'analyse sur ces phénomènes difficiles à observer de l'intérieur, mais nous pouvons rendre compte ici de ce qui a émergé explicitement dans le courant de la recherche. Ces éléments peuvent attirer l'attention sur le fait que « les chercheurs » ne constituent pas un groupe homogène du point de vue des conceptions de la recherche, des rapports aux savoirs, et de la légitimité scientifique.

Dans un premier temps, ce sont les chercheurs en sciences sociales qui sont d'emblée apparus porteurs d'un type de rapport à la scientificité différent de tous les autres (pédologues, microbiologistes, généticiens). Le chef de projet, très investi dans la production de savoirs originaux issus du partenariat entre chercheurs et producteurs, comptait sur les sciences sociales pour faciliter l'attention réflexive et transformatrice à la nature des savoirs produits au cours du programme. La sollicitation allait au-delà de la conduite d'un "volet » de recherche en sciences sociales sur la perception de l'évolution de la qualité des sols par les agriculteurs, et portait sur l'apport possible des sciences sociales au fonctionnement du partenariat de recherche et à la production de savoirs hybrides issus du partenariat. C'est cette même conception du rôle des sciences sociales produisant des

\footnotetext{
2 Le Magal de Touba est la commémoration du départ en exil de Amadou Cheik Bamba au Gabon.
} 
savoirs au statut mal défini qui peut amener certains membres des équipes de biologistes à exprimer une distance à l'égard de ces disciplines.

Mais au cours du programme les relations ont changé : les différences de conception de la scientificité qui ont amené les plus forts débats et ont mis véritablement en jeu des rapports de légitimité se sont manifestées entre les équipes de biologistes, plus précisément entre les équipes françaises de recherche en génomique travaillant avec des équipements lourds et ayant une renommée internationale, et des équipes françaises et sénégalaises en microbiologie impliquées depuis de nombreuses années dans le partenariat avec les producteurs, travaillant dans des environnements sensiblement moins instrumentés et plus articulés à des problématiques de recherche participative.

Un débat vif s'est développé dès la première année sur la qualité du travail scientifique qui était mené. Les chercheurs en génomique ont fait valoir l'importance des contraintes nécessaires à une production de données contrôlées, respectant les contraintes de temps et de coûts propres à la recherche, mobilisant de grands instruments avec l'objectif de publications internationales dans la discipline. Ils ont mis en cause les choix des microbiologistes de terrain, liés aux objectifs de la recherche partenariale (respect des dates des récoltes par exemple). Les points de vue sur les caractéristiques des savoirs à élaborer se sont avérés inconciliables et ont rendu explicites les termes d'un débat sur les rapports de légitimité scientifique entre équipes. Ce débat scientifique a fait cependant intervenir des arguments qui mobilisaient les conditions historiques et sociales de la production de sciences comme les rapports Nord/Sud dans la recherche, et surtout l'importance des conditions culturelles et sociales d'intercompréhension, explicitement rattachée à la qualité scientifique par l'une des chercheuses du laboratoire au Sénégal. Le programme a intégré dans son déroulement une radicalisation des différences de conception du savoir, pour assumer les exigences propres aux différents partenaires scientifiques, au prix d'une certaine séparation dans les opérations effectuées (notamment les prélèvements d'échantillons de terre).

Cet épisode a fait apparaître au sein du programme des tensions qui s'exercent plus largement dans la recherche en général, travaillée par des visions des sciences presque nécessairement antagonistes (production internationale d'une science de pointe dans un contexte ultra compétitif vs développement de la science participative sensible à la pertinence et aux enjeux sociaux).

\section{Conclusion}

La recherche menée a nécessité l'identification des moments et des lieux au sein desquels tous les acteurs impliqués dans la recherche se retrouvaient pour discuter ou fixer des formes du savoir collectivement élaboré, ou pour renoncer à certains consensus impossibles, à partir de positions qui rendaient les décisions légitimes.

Au-delà des processus ainsi décrits, cette démarche nous permet de dégager ce qu'est un espace des savoirs construits dans la recherche participative, espace éclaté dont les frontières ne recouvrent évidemment pas celles des «laboratoires » au sens classique. Le laboratoire de microbiologie et les lieux d'enquêtes dans les villages sont bien sûr des espaces privilégiés de légitimation des savoirs scientifiques et locaux, mais les parcelles d'expérimentation lorsqu'elles sont visitées, les ateliers délibératifs, certaines visioconférences ont constitué des espaces où se produisait et se discutait intensivement ce qu'étaient des savoirs valides.

Cet espace éclaté, constitué de tous les lieux qui rendent possibles et donnent leur sens aux situations étudiées, est pour nous une nouvelle unité d'étude des savoirs participatifs, un "laboratoire » éclaté et hétérogène mais cohérent, où l'on peut s'intéresser à des dynamiques de légitimation des savoirs et à des rapports de légitimité entre acteurs. L'identification de cet espace est en soi un résultat, issu des exigences méthodologiques et épistémologiques de penser authentiquement des conditions de production des savoirs au sein de recherches participatives, au-delà des représentations limitées que l'on peut avoir des articulations entre savoirs scientifiques et savoirs locaux, et donc de visions qui maintiennent l'idée d'un intérieur et d'un extérieur de la recherche académique.

Cet espace de la production des savoirs participatifs permet aussi de rendre compte de paradoxes apparents : le partenariat a contribué à une forme de légitimation sociale des chercheurs du projet au sein de cet espace, mais pas forcément au renforcement de leur légitimité scientifique au sein de leurs communautés académiques.

\section{Remerciements}

Nous remercions chaleureusement Sophie Lewandowski pour les discussions passionnantes qui ont alimenté certaines réflexions développées dans cet article. 


\section{Références}

Agrawal, A., 2002. Classification des savoirs autochtones : la dimension politique, Revue Internationale des Sciences Sociales, 3, 173, 325-336.

Bierschenk, T., Chauveau, J.-P., Olivier de Sardan, J.-P., 2000. Courtiers en développement : les villages africains en quête de projets, Paris, Mayence, Apad, Karthala (online: http:// empad.free.fr/IMG/pdf/Courtiers_en_developpement_ Les_villages_africains_en_quete_de_projets.pdf).

Blundo, G., 1995. Les courtiers du développement en milieu rural sénégalais, Cahiers d'Études Africaines, 35, 137, 73-99.

Boltenski, L., Thevenot, L., 1991. De la justification : les économies de la grandeur, Paris, Gallimard.

Bourguerra, L., 1993. La recherche contre le Tiers-monde : multinationales et illusions du développement, Paris, PUF.

Chambers, R., Pacey, A., Thrupp, L.A., 1989. Farmer First: Farmer Innovation and Agricultural Research, London, Intermediate Technology Publications.

Cohen, A.P., Comaroff, J.L., 1976. The management of meaning: On the phenomenology of political transactions, in Kapferer, B. (Ed.), Transaction and Meaning: Directions in the Anthropology of Exchange and Symbolic Behavior, Philadelphia, Institute for the Study of Human Issues, 87107.

Coll, J., 2000. Les courtiers en développement de MalemHodar (Sénégal), in Bierschenk, T., Chauveau J.-P., Olivier de Sardan, J.-P. (Ed.), Courtiers en développement : les villages africains en quête de projets, Paris, Apad, Karthala, Coll. Hommes et Sociétés, 101-124.

ComMod, 2005. La modélisation comme outil d'accompagnement, Natures Sciences Sociétés, 2, 13, 165-168.

Copans, J., 1980. Les Marabouts de l'arachide, Paris, Le Sycomore.

Cruise O'Brien, D.B., 1971. The Mourides of Senegal: The Political and Economic Organization of an Islamic Brotherhood, Oxford, Clarendon Press.

Ebin, V., 1993. Les commerçants mourides à Marseille et à New York. Regards sur les stratégies d'implantation, in Grégoire, E., Labazée, P. (Eds), Grands commerçants d'Afrique de l'Ouest: logiques et pratiques d'un groupe d'hommes d'affaires contemporains, Paris, Karthala, Orstom.

Ela, J.-M., 2007. Les Cultures africaines dans le champ de la rationalité scientifique, Paris, L'Harmattan.

Fassin, É., Fassin, D., 1988. De la quête de légitimation à la question de la légitimité : les thérapeutiques "traditionnelles » au Sénégal, Cahier d'Études Africaines, 28, 110, 207231.

Accepté le 4 juillet 2013.
Guèye, C., 2002. Touba, la capitale des mourides, Paris, IRD Éditions, Karthala.

Jankowski, F., 2013. Recherche participative et connaissance locale des sols au Sénégal : les typologies traditionnelles sont-elles fonctionnelles?, Revue d'Anthropologie des Connaissances, 7, 1, 271-290.

Krasova-Wade, T., Ndoye, I., 2010. Bactéries symbiotiques du sol: indicateurs des changements environnementaux? Actes du colloque international Adaptation au changement climatique: Quelles stratégies d'échange et de partage de l'information au bénéfice des communautés ?, 6-8 juillet, Dakar.

Latour, B., 1987. Science in Action: How to Follow Scientist and Engineers through Society, Cambridge (MA), Harvard University Press.

Lavigne-Delville, P., Sellamna, N.E., Mathieu, M., (Ed.), 2000. Les enquêtes participatives en débat: ambitions, pratiques et enjeux, Paris, Gret, Karthala, Icra.

Moreau, R.L, 1982. Africains musulmans, Paris, Abidjan, Présence Africaine, Inades.

Moreno Maestro, S., 2006. Le mouridisme au sein de l'immigration sénégalaise : agent de développement - Le cas de l'Andalousie, Les Cahiers du Gres, 6, 1, 93-110 (online : http:/ /id.erudit.org/iderudit/012685ar).

Mormont, M., 2007. Des savoirs actionnables, in Amoukou, I., Wautelet, J.-M., Croisement des savoirs villageois et universitaires: enjeux pour le développement, Louvain-laNeuve, Presses Universitaires de Louvain.

Oakley, P., 1991. Projects with People: The Practice of Participation in Rural Development, Geneva, International Labour Office.

Salzbrunn, M., 1996. Leaders paysans et autorités religieuses comme courtiers du développement en milieu rural sénégalais, Bulletin de l'APAD, 11 (online: http://apad. revues.org/801).

Sillitoe, P., Bicker, A., Pottier, J. (Eds), 2002. Participating in Development: Approaches to Indigenous Knowledge, London, Routledge.

Sow, H.A., Neyra, M., 2008. Paysans et chercheurs ensemble pour l'intégration des microorganismes dans le système agricole ouest-africain, Agridape, 24, 20-22.

Waast, R., Gaillard, J., 2001. La science en Afrique à l'aube du $X X I^{e}$ siècle, Paris, IRD.

Weber, M., 1959. Le Savant et le Politique, Paris, Plon.

Younes, M., 2003. Repères islamiques pour la protection de l'environnement, L'Islam Aujourd'hui, Revue périodique de l'Organisation islamique pour l'éducation, les sciences et la culture, 20. 